\title{
First accelerator test of vacuum components with laser-engineered surfaces for electron-cloud mitigation
}

Sergio Calatroni, Elisa Garcia-Tabares Valdivieso, Holger Neupert, Valentin Nistor, Ana Teresa Perez Fontenla, Mauro Taborelli, and Paolo Chiggiato CERN, 1211 Geneva 23, Switzerland

Oleg Malyshev and Reza Valizadeh

ASTeC, STFC Daresbury Laboratory, Daresbury, Warrington, WA4 4AD Cheshire, United Kingdom

Stefan Wackerow, ${ }^{1}$ Svetlana A. Zolotovskaya, ${ }^{1,2}$ W. Allan Gillespie, ${ }^{1}$ and Amin Abdolvand ${ }^{1}$

${ }^{1}$ Materials \& Photonic Systems Group, School of Science \& Engineering, University of Dundee, Dundee DDI 4HN, Scotland, United Kingdom

${ }^{2}$ Materials Science Institute, Engineering Department, Lancaster University, Lancaster LA1 4YW, England, United Kingdom

(Received 1 September 2017; published 20 November 2017)

\begin{abstract}
Electron cloud mitigation is an essential requirement for high-intensity proton circular accelerators. Among other solutions, laser engineered surface structures (LESS) present the advantages of having potentially a very low secondary electron yield (SEY) and allowing simple scalability for mass production. Two copper liners with LESS have been manufactured and successfully tested by monitoring the electron cloud current in a dipole magnet in the SPS accelerator at CERN during the 2016 run. In this paper we report on these results as well as the detailed experiments carried out on samples-such as the SEY and topography studies - which led to an optimized treatment in view of the SPS test and future possible use in the HL-LHC.
\end{abstract}

DOI: 10.1103/PhysRevAccelBeams.20.113201

\section{INTRODUCTION}

The electron cloud (e-cloud) phenomenon has long been recognized as being a major limiting factor for the beam quality of high-intensity accelerators of positive particles [1-3]. The electron multiplication phenomenon inherent in the e-cloud is directly linked to the secondary electron yield (SEY) of the surfaces of the vacuum chamber facing the particle beam. SEY thresholds have thus been evaluated for most accelerators through specialized codes [4], resulting usually in the range 1.1-1.3, lower than the SEY of typical metal surfaces used for the construction of accelerator vacuum chambers. Consequently, operational SEY mitigation techniques have been developed including the socalled "beam scrubbing" [5], where intense electron bombardment from the e-cloud results in progressively reduced SEY. Engineering solutions have also been devised and applied by design in several cases. Among those, one should mention clearing electrodes [6], weak solenoidal magnetic fields [7], thin-film coatings, in particular TiN [8] and

\footnotetext{
*Corresponding author. sergio.calatroni@cern.ch

Published by the American Physical Society under the terms of the Creative Commons Attribution 4.0 International license. Further distribution of this work must maintain attribution to the author(s) and the published article's title, journal citation, and DOI.
}

amorphous carbon (a-C) [9], topographic surface modifications either mechanical [10] or chemical [11], or a combination of these [12]. In the case of modified topography, the horizon for escape of the secondary electrons is reduced, and when impinging the nearby surfaces their low energy prevents further multiplication, thereby lowering the SEY.

In practice, topographic surface modifications are usually implemented at the manufacturing stage of the vacuum chambers. A new approach for the creation of topographically reduced SEY has been demonstrated recently [13], triggered by the success in achieving organized surface structuring of metals using robust industrial-grade short pulsed laser sources. The laser engineered surface structures (LESS) process has the advantage of being performed in air, and it can be extended to an in situ treatment inside existing accelerator vacuum chambers by implementing an appropriate beam delivery system. Motivated by this possibility, CERN has undertaken a feasibility study, in collaboration with the University of Dundee and ASTeC, in order to identify the potential of this technology for a possible application to the high-luminosity upgrade of the LHC (HL-LHC) [14].

Without mitigation measures, the energy deposited by the e-cloud on the beam screens of the focusing quadrupole magnets of the experiments in the HL-LHC will become too large compared to the local cryogenic cooling capacity [15]. New magnets will be manufactured for the interaction 
points 1 and 5 together with new beam screens, thus allowing implementing ex situ the required SEY mitigation technique, the present baseline being an a-C coating. The magnets and the screens at the interaction points 2 and 8 will instead remain unchanged, thus calling for in situ application of a mitigation treatment. Although an a-C coating can be deposited in situ, the challenges of implementing the vacuum sputtering process have suggested that the development of an alternative approach is required, in terms of implementing LESS. As an initial part of this program, we have optimized the laser engineering parameters on copper through sample studies, and then implemented LESS on two copper liners whose performance in terms of e-cloud intensity has been assessed in an accelerator for the first time. In this paper, we discuss in detail these sample studies and the experimental measurements on the liners carried out with an e-cloud monitor (ECM) in the SPS accelerator.

\section{EXPERIMENTAL SETUPS}

\section{A. Sample preparation and SEY characterization}

Samples were prepared out of 1-mm thick Cu-OFE sheets, cut into strips of $120 \mathrm{~mm} \times 20 \mathrm{~mm}$ for convenience, degreased and passivated at CERN following standard procedures prior to shipping for laser treatment. All strips were laser treated either at the University of Dundee or by ASTeC, and shipped back to CERN wrapped in silk paper and a layer of aluminum foil, then packaged in PE bags backfilled with nitrogen. The time between sample laser treatment and SEY measurement was usually of the order of a couple of weeks.

SEY measurements at CERN have been performed using the facility described in detail elsewhere [16]. The measurements are performed in UHV, and the samples are inserted in the measurement chamber through a load-lock system. Typical sample size is $15 \mathrm{~mm} \times 20 \mathrm{~mm}$, and the measurement is performed usually on five different spots of about $2 \mathrm{~mm}^{2}$ in order to assess reproducibility of the treatment. The measurements proceed typically by scanning the energy of the primary electrons, which impinge on the surface at normal incidence, from 50 to $1750 \mathrm{~V}$ while measuring simultaneously the currents to the sample and to the secondary electrons collector, from which the SEY is calculated. The precision of the measured SEY values is estimated at \pm 0.05 . The total dose delivered during the measurement of one SEY curve like those reported in the next section is about $10^{-6} \mathrm{C} \mathrm{mm}^{-2}$, which is low enough to prevent any conditioning during the measurements. No surface charging was detected either, each measurement curve being reproducible within the experimental uncertainty.

\section{B. Liner preparation and SPS test setup}

The present experiments were conducted in two of the four ECM test stations available in the SPS accelerator, described in detail in $[9,17]$ (Fig. 1). Each test station comprises a so-called C-magnet, usually set to produce a dipole field of $0.12 \mathrm{~T}$ equal to the SPS injection field, enclosing a round vacuum chamber into which the liners are inserted. The liners for the SPS accelerator test were manufactured from $\mathrm{Cu}-\mathrm{OFE}$ sheets. They are $1030 \mathrm{~mm}$ long and have a cross section similar to that of the main bending type $\mathrm{B}$ dipole chambers (MBB) of the SPS, where the e-cloud phenomenon is the most severe. The top face of the liners has a series of $2 \mathrm{~mm}$ diameter holes distributed over a total surface of $380 \mathrm{~mm} \times 110 \mathrm{~mm}$, with a total transparency of about 7\%, allowing the electrons of the cloud to escape and be collected by the multistrip electron current monitor. The laser treatment was limited to two mirroring areas on the top and bottom inner faces of the liner, sufficiently wide transversally to encompass the entire e-cloud and extended longitudinally beyond the perforated region, see Fig. 2(a). Since the dipole magnetic field lines confine the e-cloud phenomenon locally, electrons generated in the untreated regions are not collected. The laser treatment has been performed at the University of Dundee and by ASTeC on the open top and bottom halves of the
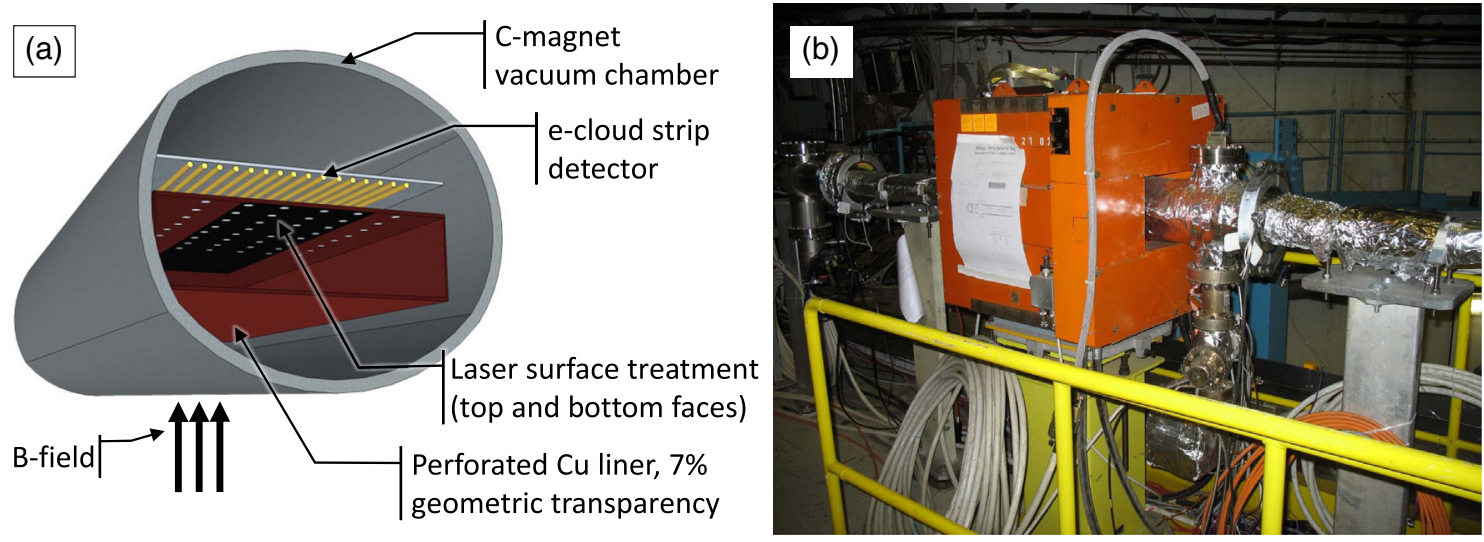

FIG. 1. (a) Schematics of the ECM system. (b) Assembly of the C-magnet in the SPS tunnel. 

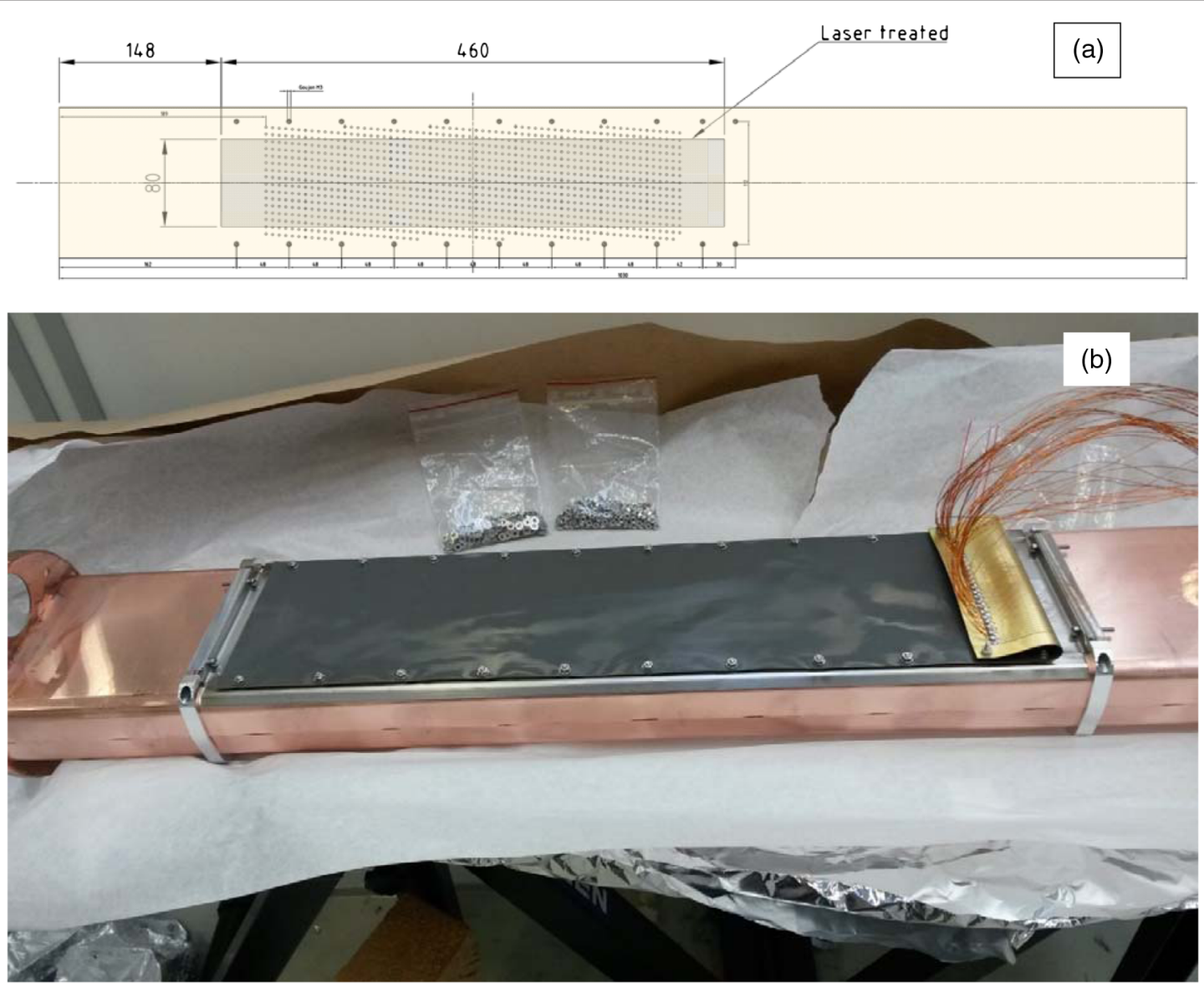

FIG. 2. (a) Schematics of the laser treated region on the top half of the liner; the bottom half is treated symmetrically. (b) One of the finished liners assembled with the stripline monitor before installation in the SPS magnet.

liners, shipped to CERN for their final assembly performed by spot welding. The finished liners have been degreased with alkaline detergents following the standard CERN UHV procedure for copper.

The multistrip electron detector is assembled outside the liner in correspondence with the perforated region and comprises 48 longitudinal pickup copper strips supported on a polyimide foil, positively polarized and which can be read individually. Prior to assembly on the liners, the electron detector is baked in vacuum at $120^{\circ} \mathrm{C}$ for 24 hours, in order to partly degas the water absorbed by the polyimide. One final assembly is illustrated in Fig. 2(b).

\section{Laser treatment process}

Different lasers treatment procedures have been tested in the two SPS liners, after a substantial development work on samples.

A number of test samples were produced for initial studies at the University of Dundee with subsequent comprehensive surface characterization performed at CERN. The laser surface structuring was carried out using a linearly polarized 10 -ps laser beam with a wavelength of $532 \mathrm{~nm}$ at a repetition rate of $200 \mathrm{kHz}$. The laser beam had a Gaussian intensity profile $\left(\mathrm{M}^{2}<1.3\right)$ and was focused onto the copper target surface using a flat-field scanning lens system-a specialized lens system in which the focal plane of the deflected laser beam is a flat surface-equipped with a telecentric F-theta lens. These arrangements allowed for offsetting the off-axis deflection of the beam through the focusing lens system. The diameter of the focused spot-between the points where the intensity has fallen to $1 / e^{2}$ of the central value-was measured to be $\sim 12 \mu \mathrm{m}$ leading to a laser beam intensity of $\sim 0.4 \mathrm{TW} \mathrm{cm}^{-2}$ in the focus. Throughout the experiments, an average laser pulse energy of $\sim 5 \mu \mathrm{J}$ was used, leading to a laser energy fluence of $\sim 4.2 \mathrm{~J} \mathrm{~cm}^{-2}$. This value is well above the experimentally measured value for the ablation threshold of copper at $532 \mathrm{~nm}$ of $\sim 0.42 \mathrm{~J} \mathrm{~cm}^{-2}$, but is well below the assessed thermal threshold of copper at $532 \mathrm{~nm}$ of $\sim 10.6 \mathrm{~J} \mathrm{~cm}^{-2}$. These were determined experimentally using the self-consistent evaluation of the Gaussian beam diameter and the ablation threshold as described in [18].

For the preliminary experiments, the laser beam was raster scanned over the surface of the target at $10 \mathrm{~mm} \mathrm{~s}^{-1}$, irradiating $2 \mathrm{~mm} \times 2 \mathrm{~mm}$ areas using a computer-controlled scanner system. Two different scanning techniques were employed for these experiments, namely cross hatched $(\mathrm{CH})$ 

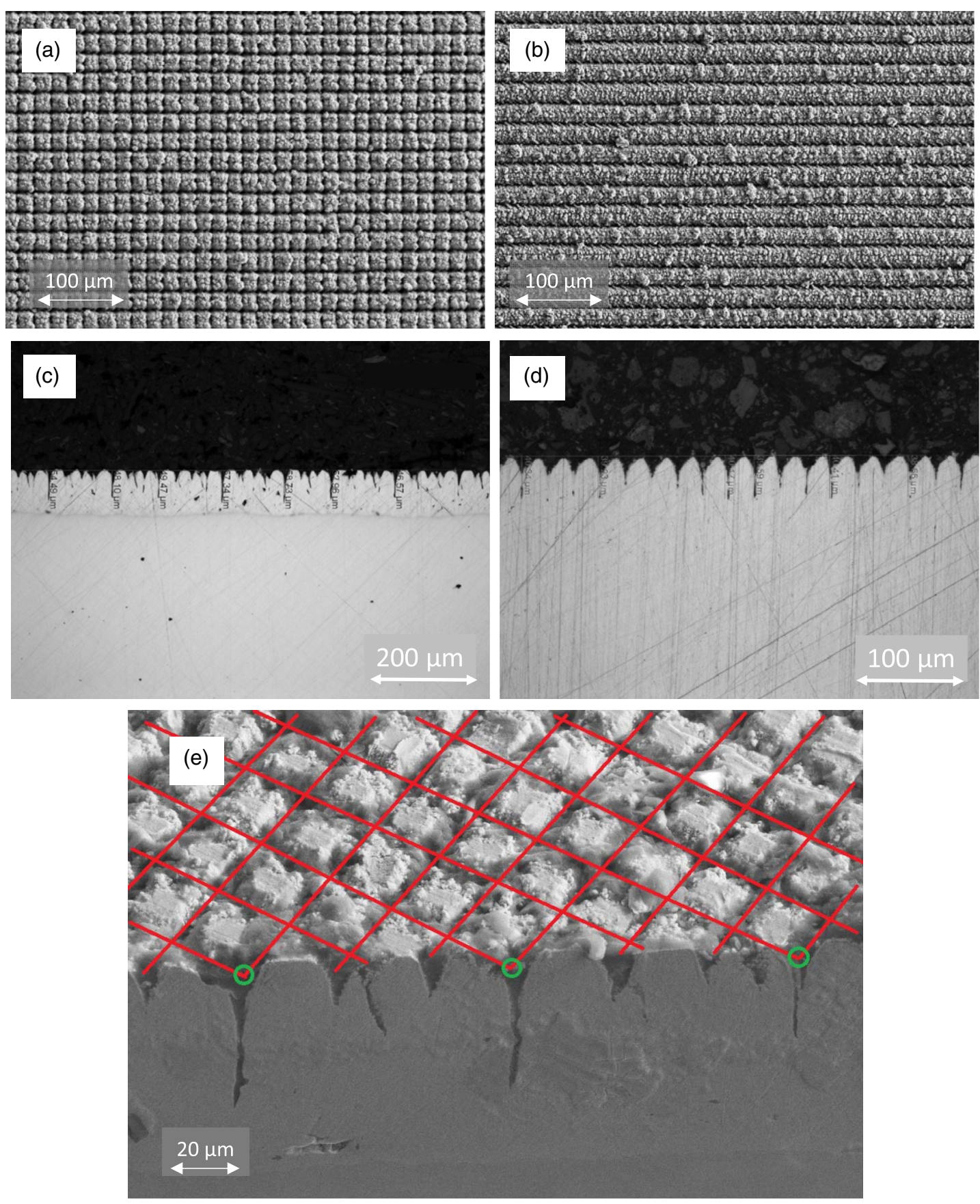

FIG. 3. (a) SEM top view of a $\mathrm{Cu}$ sample treated with $\mathrm{CH}$ pattern and (b) $\mathrm{LH}$ pattern at the University of Dundee. (c) Optical microscope image of a mechanically cut and polished cross section of (a) where the average depth of the grooves is estimated at $53 \pm 5 \mu \mathrm{m}$ and (d) cross section of (b), where the average depth of the grooves is estimated at $35 \pm 6 \mu \mathrm{m}$. (e) SEM cross-section image illustrating the larger ablated depth at crossing patterns.

scanning, shown in Fig. 3(a), and line hatched (LH) scanning, shown in Fig. 3(b). For the LH pattern the laser was scanned only in one direction, while for the $\mathrm{CH}$ pattern the laser was scanned over the surface in two perpendicular directions to form a grid pattern, with the total number of pulses fired onto the target surface being twice as many as in the LH pattern, thus doubling the time required for treatment. During each scan in the LH patterning approximately
240 pulses per spot were fired onto the target. Using the laser parameters employed here, the theoretical assessments based on the 1D thermal diffusion model for picosecond pulsed laser ablation [19-21] have led to an $\sim 180 \mathrm{~nm}$ ablation depth per laser pulse. Therefore, the experimentally assessed ablation depth of (35 $\mu \mathrm{m} \pm 6 \mu \mathrm{m}$ ) in Fig. 3(d) for the LH pattern is in good agreement with the theoretically predicted value of $\sim 43 \mu \mathrm{m}$. It has to be pointed out that, 
although in the $\mathrm{CH}$ patterning approximately 480 pulses per spot are fired onto the target at the crossing of the patterns, the experimentally observed ablation depth of $(53 \mu \mathrm{m} \pm 5 \mu \mathrm{m})$ in Fig. 3(c) is indeed expected, rather than higher values. This is due to the fact that in the $\mathrm{CH}$ pattern and during scanning of the surface in the second direction, the laser beam faces an uneven surface that has already been modified by the first scan. Hence, a proportion of the optical energy is deflected by the structures produced during the first scan. This prevents further penetration of the laser beam into the material. Figure 3(e) clearly illustrates the larger ablated depth at the line crossing.

A scanning electron microscope (SEM) image of the cross section of a sample analogous to Fig. 3(b), cut with a focused ion beam (FIB), is illustrated in Fig. 4. From these images one can clearly distinguish a first region visible immediately above the bulk $\mathrm{Cu}-\mathrm{OFE}$ and roughly estimated in hundreds of nanometers of thickness which presents close porosity and where the material seems to be adherent to the bulk, indicated by the red rectangle in the figure. A second region, indicated by a green rectangle, of an estimated thickness of a few microns and in direct contact with the bulk $\mathrm{Cu}-\mathrm{OFE}$ or over the first mentioned region, presents a lower density and open porosity with cavities of varying size, made of particles that are only slightly attached to each other or to the bulk copper.
The liner at the University of Dundee was structured in house in air at atmospheric pressure using the above laser parameters. The LH patterning was selected for this liner due to the smaller laser beam penetration depth during the interaction. This is also motivated by the fact that the LHC beam screens are fabricated with a special high-Mn stainless steel alloy (P506) co-laminated with an $80 \mu \mathrm{m}$ thick $\mathrm{Cu}-\mathrm{OFE}$ layer, setting the maximum depth of the possible surface structuring at about one-half of this value. The lines of structures were produced along the length of the liner, i.e. parallel to the beam axis. The employed laser parameters resulted in only small loose clusters (less than $1 \mu \mathrm{m}$ in diameter) being generated during the structuring process that were extracted using a laser fume extractor equipped with a filter throughout the laser surface structuring experiment. In order to cover the envisaged area of $460 \mathrm{~mm} \times 80 \mathrm{~mm}$ on each half of the liner [Fig. 2(a)], the lens patterning area was set to $40 \mathrm{~mm} \times 46 \mathrm{~mm}$, adjusting the laser focus at the center of each square. Two rows of ten squares were laser structured on each half of the liner [see Fig. 6(a)], taking approximately 80 hours of total processing time for the liner. No pressurized nitrogen flow was used to remove dust prior to shipment.

A comprehensive study on the laser ablation process parameters was also carried out by ASTeC. Most of this development work was performed using a $355 \mathrm{~nm}$ laser of

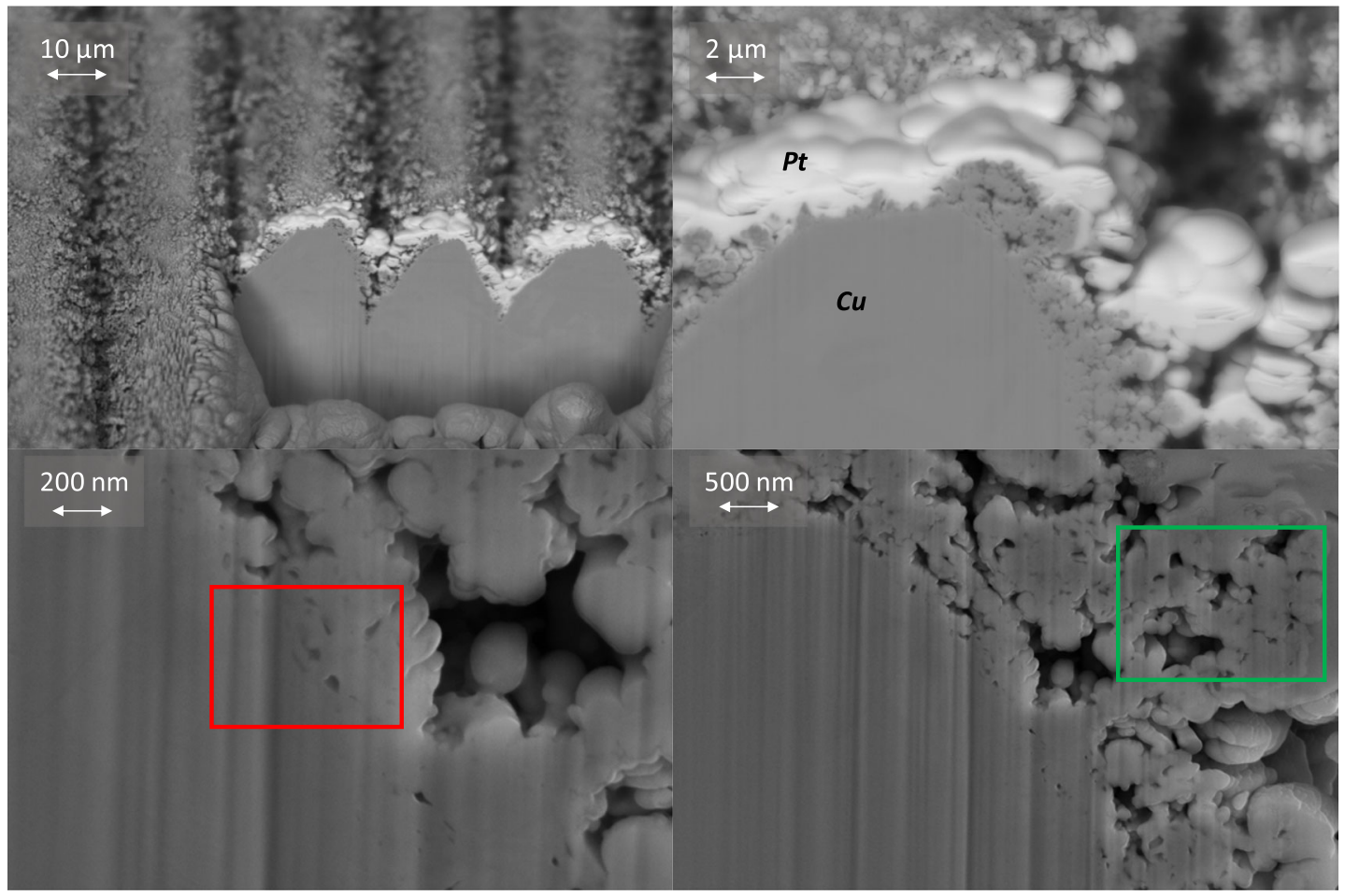

FIG. 4. Comprehensive view of a FIB cross section of a LH sample analogous to Fig. 3(b), at progressive magnifications clockwise from top left. The platinum top layer, of lighter color in the images, is added as a part of the FIB etching process, to improve image quality minimizing artifacts. The colored rectangles indicate the different regions mentioned in the text. 

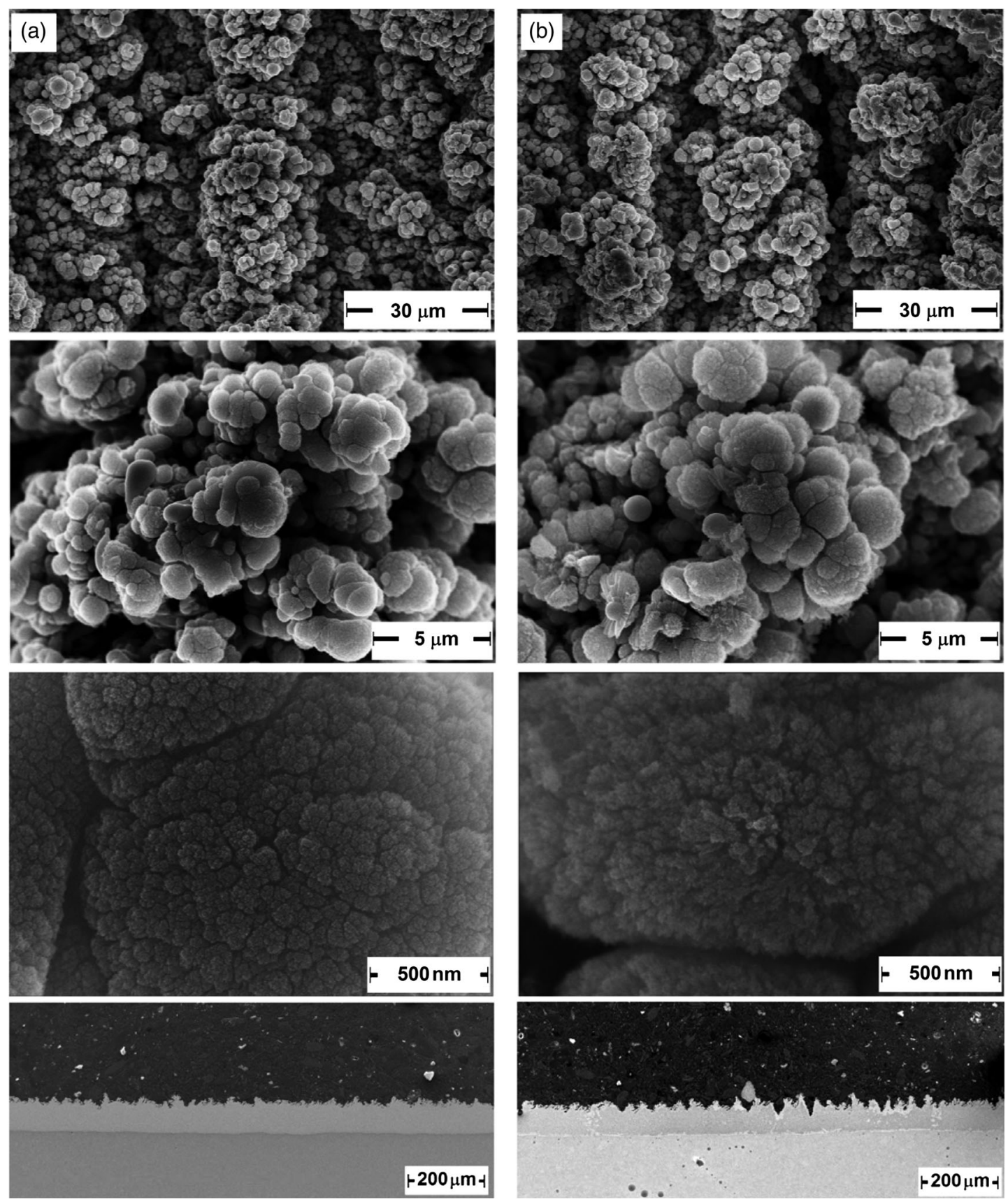

FIG. 5. SEM images of test samples treated with LH pattern by ASTeC at $120 \mathrm{~mm} / \mathrm{s}$ (a) and $90 \mathrm{~mm} / \mathrm{s}$ (b) showing an hierarchy of surface structures at different magnifications on top views (three top rows) and cross section views (bottom row).

$3 \mathrm{~W}$ average power, with a pulse duration of $25 \mathrm{~ns}$ at repetition rate of $40 \mathrm{kHz}$ (75 $\mu \mathrm{J}$ per pulse). This results in a fluence of $42 \mathrm{~J} \mathrm{~cm}^{-2}$ for a spot diameter of $15 \mu \mathrm{m}$ at $1 / e^{2}$ Intensity. This work is discussed and summarized in detail in [22], where several scanning speeds and pitches were tested.

Based on these results, two sets of laser parameters were identified as most suitable for accelerator application, namely those with scan speeds of 90 and $120 \mathrm{~mm} / \mathrm{s}$ (see Fig. 5) with an LH pattern at $10 \mu \mathrm{m}$ scanning pitch, which result in groove depth of 35 and $20 \mu \mathrm{m}$ respectively. These represent different compromises among the maximum depth of the treatment, the resulting SEY and the rf surface resistance [23].

The liner by ASTeC was laser treated following the ASTeC defined parameters at Micronanics Laser Solution Centre (RAL, Oxfordshire, UK) also in air at atmospheric pressure. To pattern the desired area on each half of the liner an F-theta scan lens was used. The error in flatness was compensated for by first leveling the liner surface and then mapping the surface and using a correction table to correct each site for focus changes. Due to the 


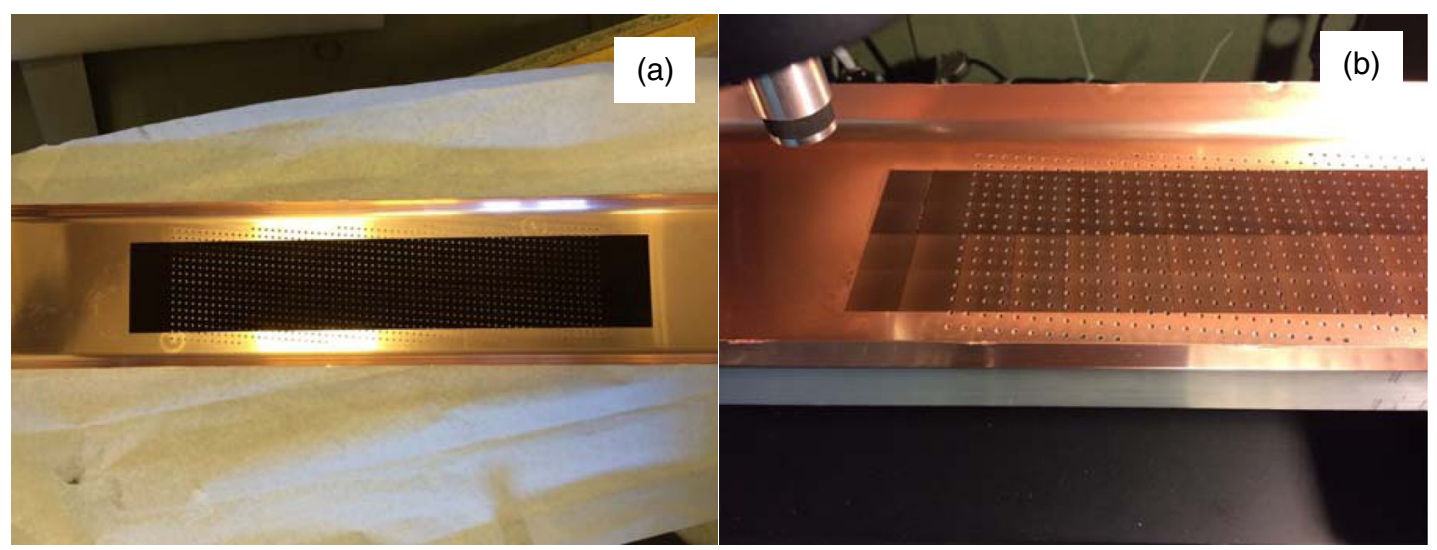

FIG. 6. Perforated side of the liners treated (a) at the University of Dundee, and (b) by ASTeC. In (b), the two regions treated with a different set of laser parameters are clearly visible, the darker area having been treated at $90 \mathrm{~mm} / \mathrm{s}$.

restricted patterning area of the used lens, an area of $20 \mathrm{~mm} \times 20 \mathrm{~mm}$ was scanned first. This was repeated for two rows covering an area of $460 \mathrm{~mm} \times 40 \mathrm{~mm}$ with a scan speed of $90 \mathrm{~mm} / \mathrm{s}$, with the grooves parallel to the beam axis. The scan speed was then increased to $120 \mathrm{~mm} / \mathrm{s}$ and a further two rows processed, on the adjacent region symmetric to the liner axis. Due to the locality of the ecloud in a dipole magnetic field, it is in fact possible to treat regions of the liner which are laterally symmetric with respect to the beam axis with different laser parameters, and measure any difference in e-cloud performance within one single experiment [9].

The other half of the liner was treated performing a mirror image so that the areas treated at $120 \mathrm{~mm} / \mathrm{s}$ scan speed would be top and bottom of the same half of the tube, likewise with the areas treated at $90 \mathrm{~mm} / \mathrm{s}$ scan speed [see Fig. 6(b)]. The total process time was also of the order of 80 hours.

All laser treatments were carried out in a class 3 clean room, and a filtered extraction was used to collect as much of the debris produced during the ablation processing as possible. Furthermore, pressurized nitrogen flow was used to remove dust prior to shipment.

\section{RESULTS AND DISCUSSION}

\section{A. SEY}

All laser structuring experiments discussed here were performed in air at atmospheric pressure. Typical SEY curves for samples cut from strips treated at the University of Dundee are reported in Fig. 7, for $\mathrm{CH}$ (green curve) and LH (red curve) patterns, averaged over five sampling locations each. Both show SEY lower than 1, which would result in full suppression of any electron multipacting phenomenon in a particle accelerator. This result, coupled to the strong reduction in the depth of the etched trenches and the obvious reduction of a factor 2 of the time needed for treatment, motivated the choice of the LH pattern for treating the SPS liner.
A sample from the same LH production batch, kept for almost one year at CERN in air wrapped in the original packaging, has been measured with the goal of verifying aging. The result is reported in Fig. 8 (plotted in purple), together with the SEY curve of a typical pristine sample (in red). The SEY degrades by less than 0.1 , indicating a very good stability of the laser treatment.

As reconfirmation of the results published in [22], SEY measurements have been performed at CERN also on samples from ASTeC produced at 90 and $120 \mathrm{~mm} / \mathrm{s}$. These are reported in Fig. 9. Although the SEY curve is slightly higher than that of the samples from the University of Dundee, SEY is below 1 up to primary electron energies of about $1 \mathrm{keV}$, well above the typical electron energies in the e-cloud [3].

It should be noted that the trenches observed for example in the LH pattern in the SEM images of Fig. 3 take at most $20 \%$ of the sample area. Even if no secondary electrons were at all emitted from these areas, this would not be

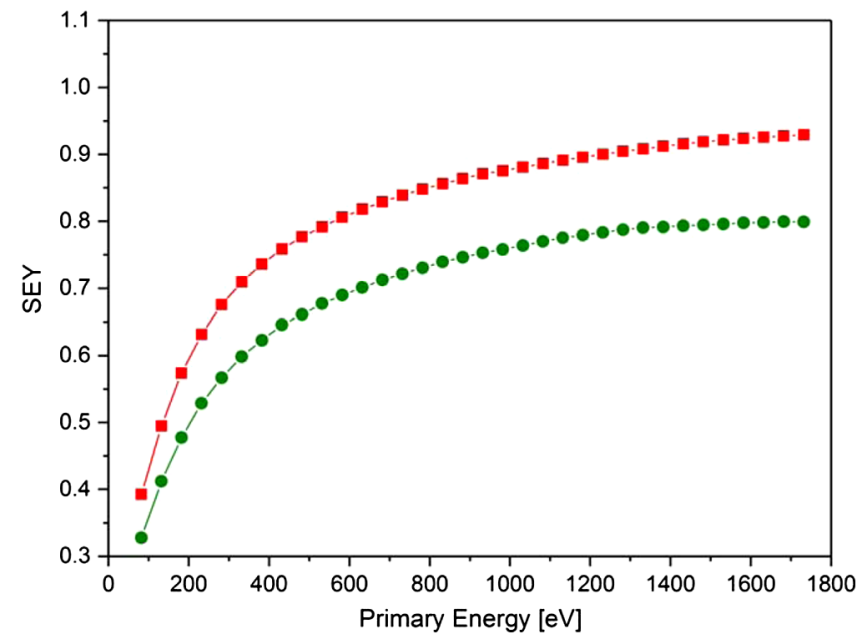

FIG. 7. SEY comparison of $\mathrm{CH}$ (green circles) vs LH (red squares) patterns for samples from the University of Dundee. 


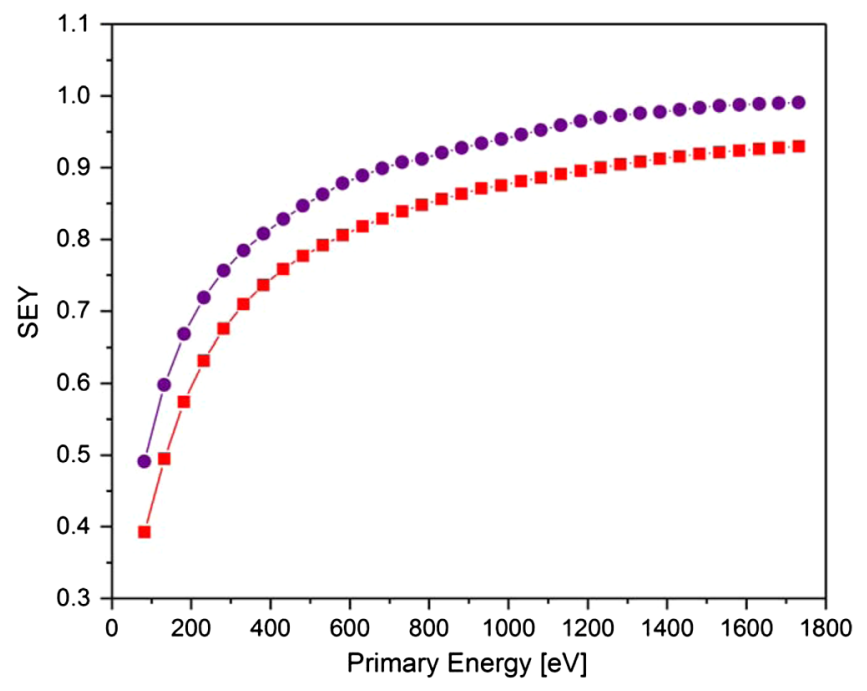

FIG. 8. Secondary electron yield of a University of Dundee LH sample as received (red squares) and after 12 months of aging (purple circles).

sufficient to explain a reduction of SEY from the value of $\sim 2$ typical of unconditioned copper [24] to values lower than 1 . Thus, it is plausible to deduce that the dominant role in reducing SEY is played by the fine copper structures on the surfaces between the trenches produced by redeposited metal from the ablation process, and not by the trenches themselves, as can be evinced also from Fig. 5. The lower SEY resulting from the $\mathrm{CH}$ pattern could then be ascribed to the larger amount of this fine structure observed in the SEM images, due to the doubling of the ablation.

We also carried out measurements of the pressure decrease versus time, the so-called pumpdown curves, of different sets of strips laser treated at the University of Dundee and inserted in a dedicated vacuum system, pumped with a turbomolecular (TM) pump. In this regime,

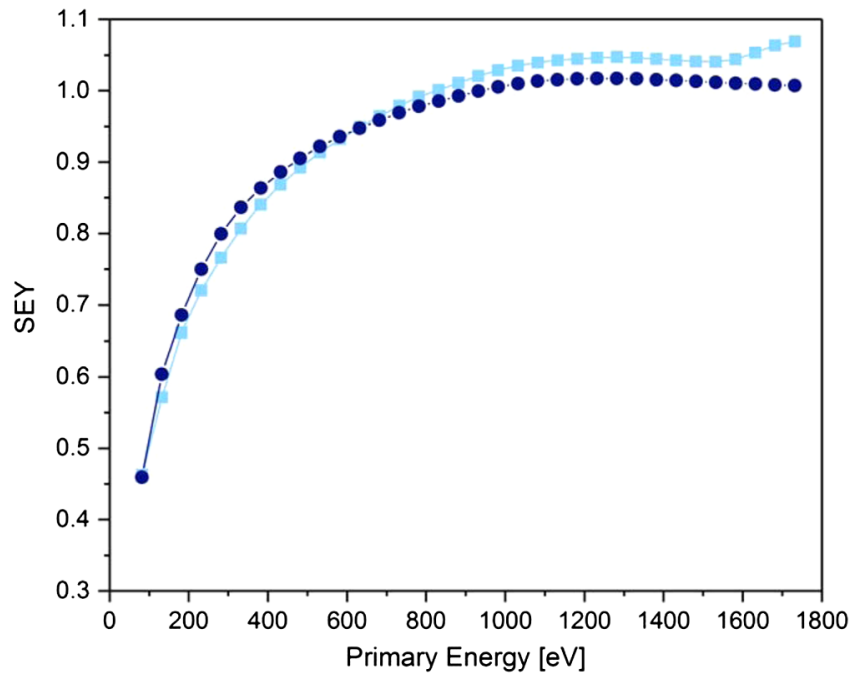

FIG. 9. SEY of samples from ASTeC treated at $90 \mathrm{~mm} / \mathrm{s}$ (dark blue circles) and $120 \mathrm{~mm} / \mathrm{s}$ (light blue squares) with LH pattern. the measured water outgassing is typically attributed to the effective surface area, as compared to the geometric area of a sample. Using the standard criterion of measuring the water outgassing after ten hours of pumping, laser-treated samples showed outgassing values of the order of 100 times larger than for plain copper, which can be ascribed to the presence of the fine copper structures on the surface. The large uncertainty inherent in this measurement did not allow identifying any major differences between LH and $\mathrm{CH}$ laser patterns. Although the large water outgassing could certainly be a limit for some applications, it is not expected to have a major impact in our case, due to the limited area treated compared to the surrounding environment, as will be discussed later.

As mentioned earlier, the liner fabrication process required a final degreasing including ultrasonic agitation
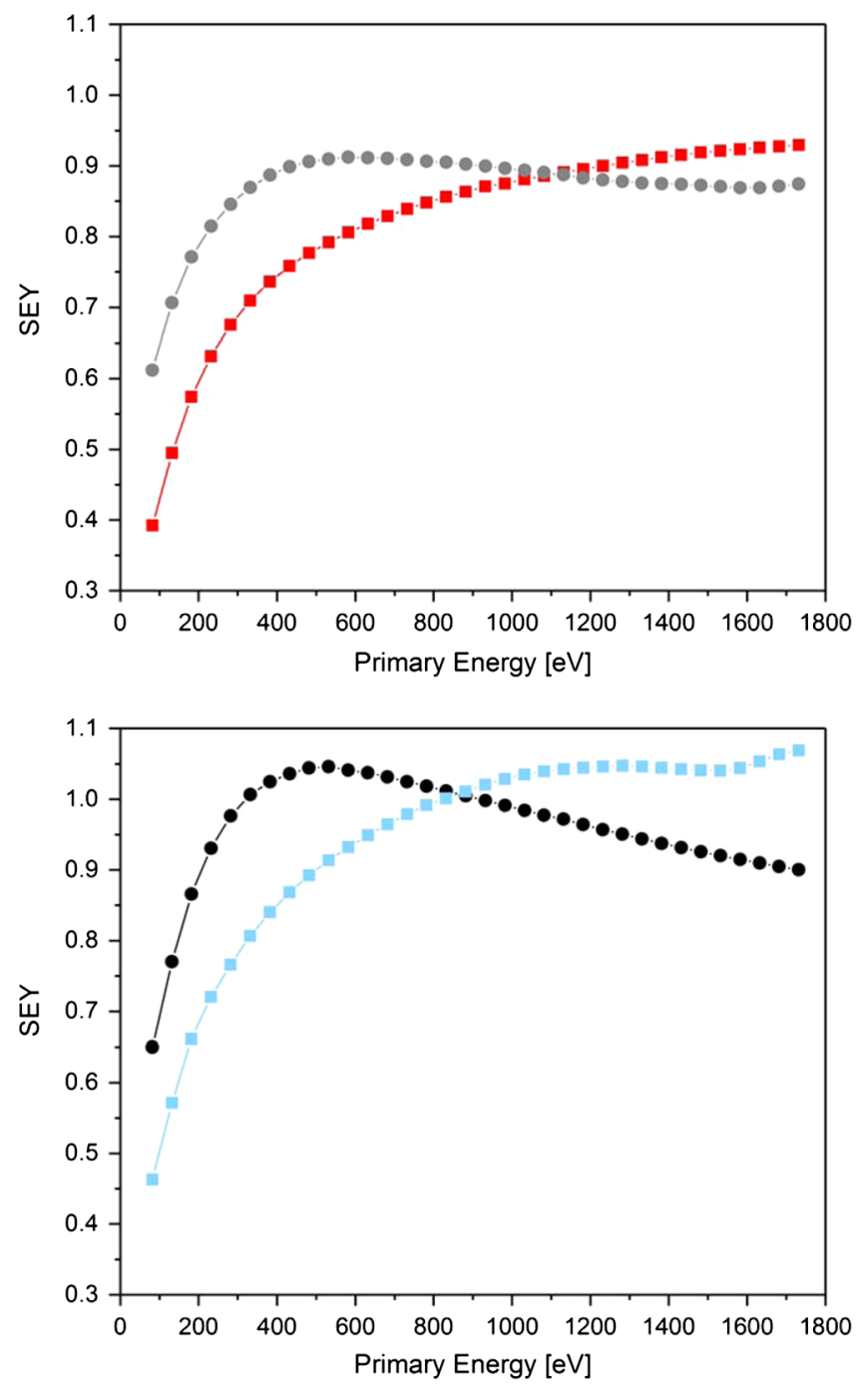

FIG. 10. SEY curves for: (top) LH samples produced at the University of Dundee before (red squares) and after (grey circles) the CERN standard cleaning procedure; (bottom) LH $120 \mathrm{~mm} / \mathrm{s}$ samples from ASTeC before (light blue squares) and after (black circles) the same cleaning procedure. 
following the standard CERN procedure for copper in order to guarantee surface cleanliness compatible with UHV. This might damage the surface and result in a change of SEY because of topography changes. An experiment has thus been performed on samples provided both by the University of Dundee and ASTeC, measuring SEY after a standard degreasing cycle. The results are shown in Fig. 10, where a clear change in the shape of the SEY curve can be seen, namely a reduction of the SEY peak energy $\mathrm{E}_{\max }$. On a rough surface, the average incidence angle at which the primary electrons encounter the material is larger than for normal incidence on a flat surface, and as a consequence the effective penetration depth of the primary electrons in the material decreases. This favors the escape of the secondary electrons up to a larger primary energy than for normal incidence and therefore the $\mathrm{E}_{\max }$ increases with roughness. In the present case, removing part of the roughness by the ultrasonic treatment brings the samples back in the direction of a flat surface, and the $\mathrm{E}_{\max }$ decreases.

As an example, the surface modification resulting from the cleaning process is clearly visible from the SEM images in Fig. 11, performed on a $\mathrm{CH}$ sample from the University of Dundee. We should also note that neither surface modification nor visible particle detachment was observed when blowing the surface of a reference sample with a common laboratory nitrogen gun, fed at 3 bar. On the other hand, particles up to $3 \mu \mathrm{m}$ in size were observed to detach from the surface when sampling it with adhesive tape and analyzing the collected material under the SEM.

Although the peak values of SEY are here close to unity, the e-cloud threshold calculated for the SPS liners is of the order of 1.3 [25], thus full e-cloud suppression is expected from the SPS experiment.

\section{B. SPS results}

Two liners have been treated following the procedures discussed above, and installed in the SPS accelerator during the Year-End Technical Stop 2015-2016 in the ECM test stands \#1 (treated at the University of Dundee) and \#4 (treated by ASTeC). A standard copper liner was already installed in one of the other test stations (\#2) to serve as a reference, while test station \#3 was still equipped with an a-C coated copper liner, installed in the 2007-2008 accelerator winter stop and kept in the machine for a longterm study.

Pumpdown of the vacuum sector containing the four test stations was carried out following SPS standard procedures, by first evacuating with TM pumps until pressure reaches the range of $10^{-6}$ mbar, when ion pumps are turned on. These are then kept on during machine operation, after disconnecting the mechanical pumping stations. Pressure decrease curves in this particular accelerator sector are strongly influenced by all the other accelerator components which are present, thus no information on the water outgassing from the liners surface could be deduced. This is in line with expectations, given the limited amount of treated surface compared to the size of the vacuum sector, and similar to what was observed with a-C coated liners [9].

An accelerator test run of four hours, a so-called machine development (MD) session, took place in June 2016solely dedicated to this study - the operation of the dipole field in the ECM test stations being incompatible with normal operation or other machine studies. The chosen beam conditions for the measurement follow common practice for this type of studies [9]: $26 \mathrm{GeV}$ proton beams (no acceleration) of four batches of 72 bunches each (288 total) with a bunch spacing of $25 \mathrm{~ns}$ and a bunch charge of $1 \times 10^{11}$ protons. The beam is circulating for
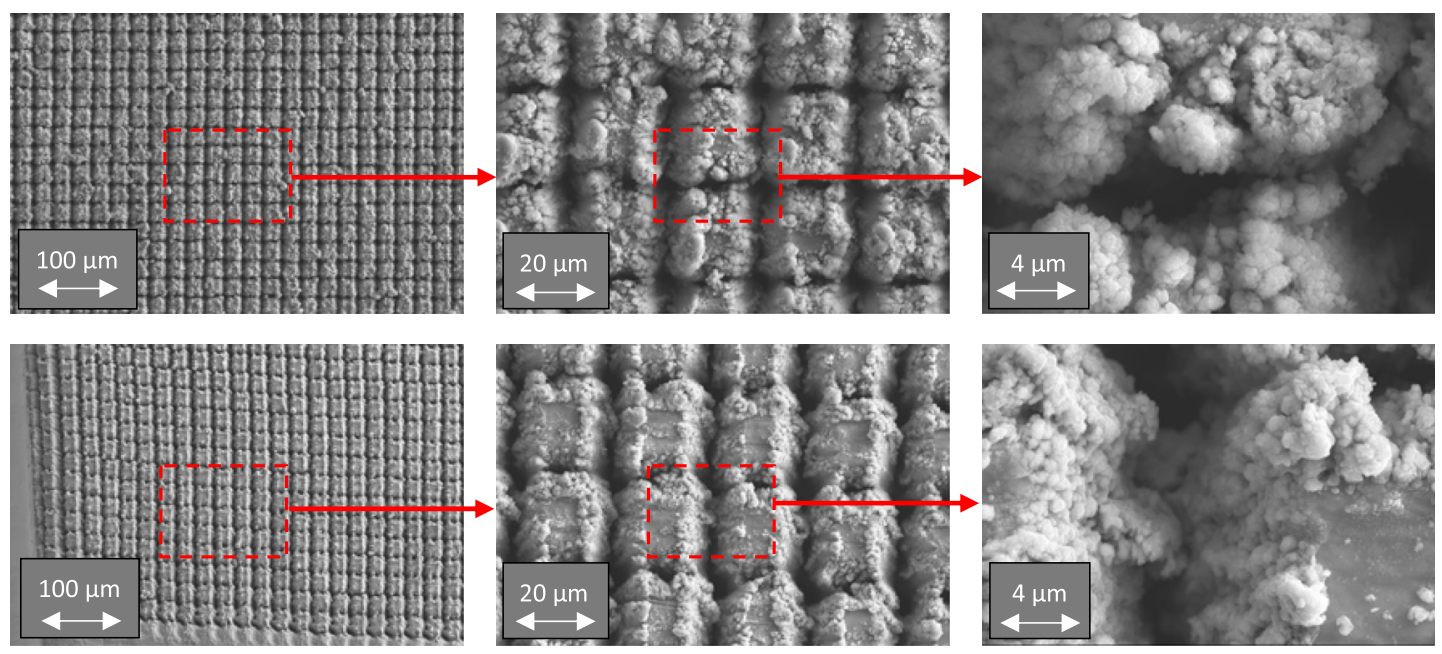

FIG. 11. SEM images at different magnifications of a $\mathrm{CH}$ reference sample produced at the University of Dundee (top row) and of a twin sample after cleaning with ultrasonic agitation for 5 minutes (bottom row). 
approximately $20 \mathrm{~s}$, after which it is dumped and a new beam is injected again in a global cycle of $24.5 \mathrm{~s}$. The bunch population and thus the average machine current has been stable within a few percent throughout the duration of the experiment.

The signals of the 48 strips of the detectors have been summed and integrated over the duration of each machine cycle, and each of the resulting data points is plotted as a function of time during the experiment in Fig. 12. Both laser-treated liners, as well as the a-C coated liner, show an electron signal which is about 4 orders of magnitude smaller than the reference copper liner. Copper shows clear signs of conditioning during the experiment as expected, the signal reducing by more than a factor 2 but still remaining much stronger than in the liners treated with SEY mitigation techniques. Some intensity difference is seen among the three liners having e-cloud mitigation treatments, correlated with the measured SEY. A minor trend of improvement can also be seen in one of the laser treated liners and in the a-C coated one. However, in all cases the SEY is expected to be well below the electron multiplication threshold and thus the measured signal should come only from primary electrons. At these low intensities, it is possible that the measured differences could then arise from experimental artifacts. In Fig. 13 we report the sum of the current of the 48 channels of each detector plotted against time during $25 \mathrm{~s}$, thus covering a representative accelerator cycle. The signal measured in the laser treated and a-C coated liners appears to be largely dominated by random noise having both positive and negative values. The integration of this signal, which would result in one data point in Fig. 12, should thus be assumed compatible with zero within experimental uncertainty. On the other hand, in the case of the untreated copper liner, one can clearly identify the injections of each of the four batches of 72 bunches, and the consequent progressive development of the e-cloud. We should also note that as expected we saw no differences between the two sides of the liner treated by $\mathrm{ASTeC}$, which were treated with different laser parameters, when integrating and comparing the two sets of 24 channels on each side.

The suppression of the e-cloud phenomenon is usually associated with an improvement of dynamic vacuum in operation. However, as discussed in [9] for a-C coated liners, the large conductance of the vacuum chambers does not allow in this test to put in evidence any correlation between SEY reduction and vacuum levels, the latter being largely dominated by the untreated machine parts.

The laser-treated liner installed in the ECM test stand \#1 will be kept in the SPS accelerator with the goal of performing long-term studies. The measurement has
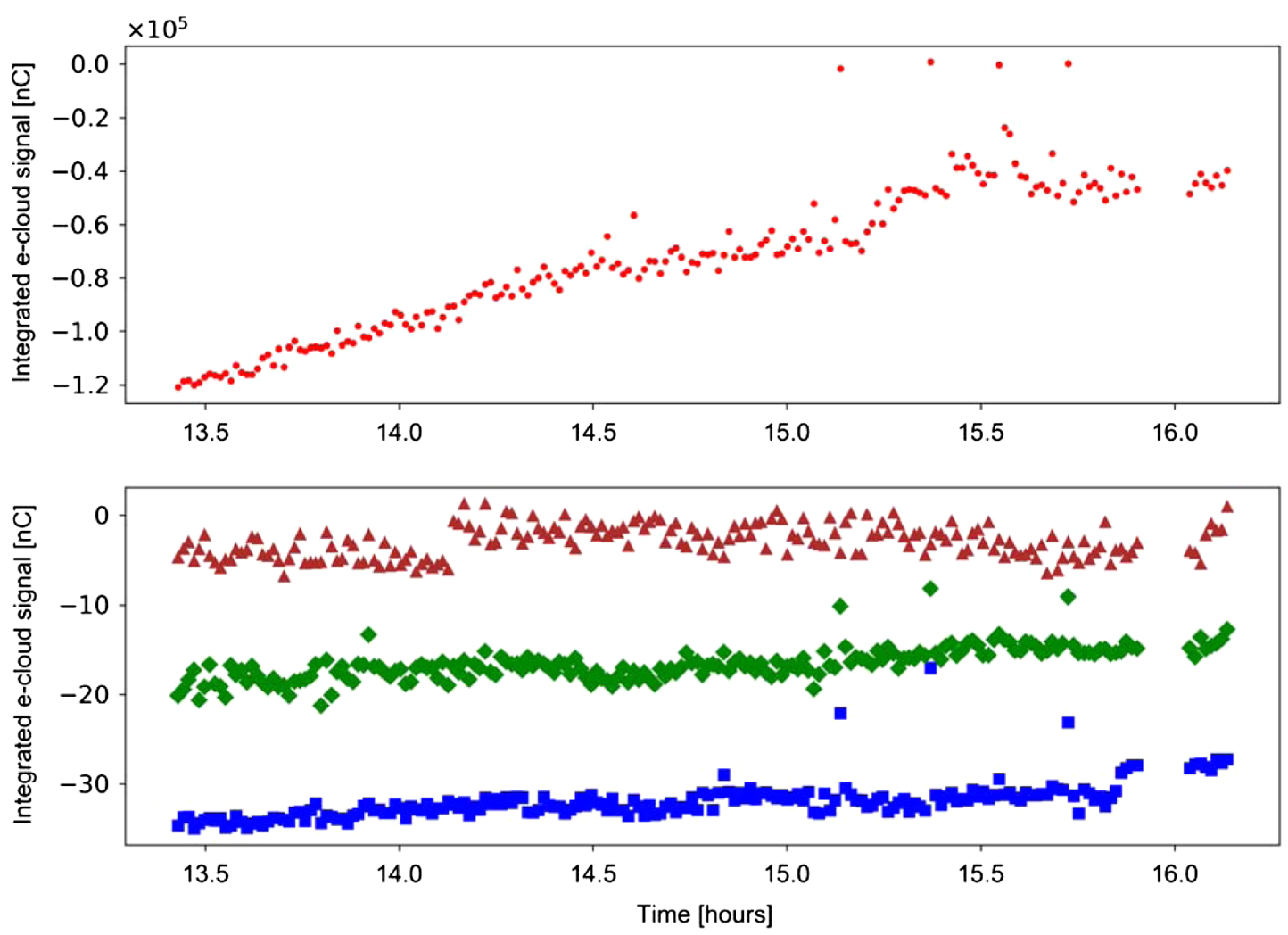

FIG. 12. Integrated signals from the ECMs during the total duration of the experiment in the SPS. (top) Reference copper liner; (bottom) liners with e-cloud mitigation: blue squares - treated by ASTeC, brown triangles - treated at the University of Dundee and green lozenges - a-C coating. Note the difference in vertical scales. 


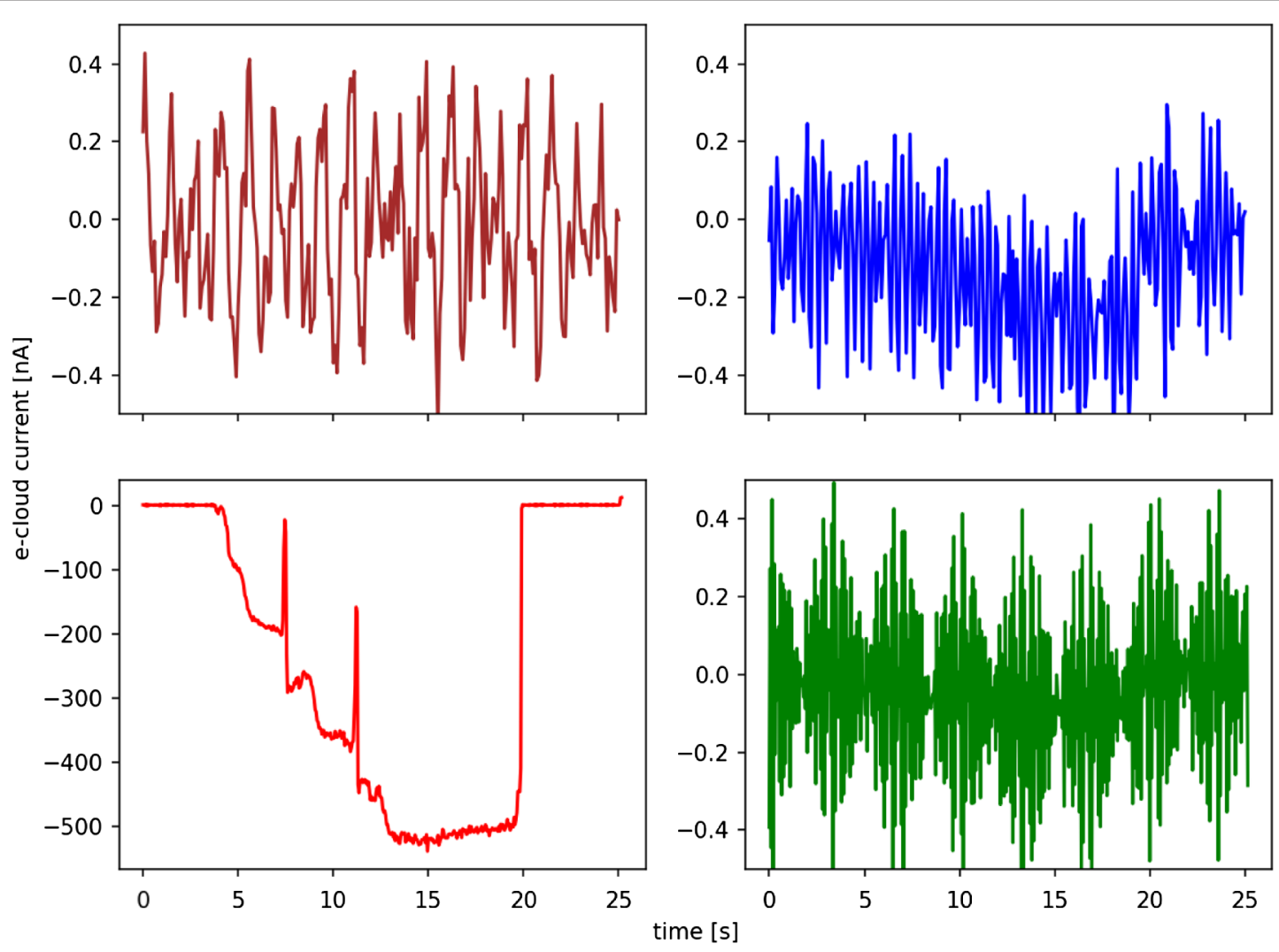

FIG. 13. Progressive e-cloud development in the four batches of 72 proton bunches. Clockwise from top left: liner treated at the University of Dundee, liner treated by $\mathrm{ASTeC}$, a-C coated liner and $\mathrm{Cu}$ liner. Note the difference in vertical scale for the latter.

already been repeated after one year, during an analogous MD session performed in July 2017 with similar beam parameters, reproducing the results obtained earlier. This confirms the stability of the laser treatment over time for suppressing e-cloud, as was already anticipated by the SEY results shown in Fig. 8.

The SPS test successfully proves the total suppression of the e-cloud phenomenon, as was anticipated from the measured SEY values, thus giving a strong motivation for further study of this technique in view of its implementation for the HL-LHC.

\section{CONCLUSIONS AND OUTLOOK}

Electron cloud mitigation based on LESS has been successfully demonstrated for the first time in a particle accelerator. Extensive development work on test samples through optimization of the laser treatment parameters and the SEY, demonstrated that SEY $<1$ can be consistently achieved for practical implementations on technical surfaces. This allowed treating two 1-m copper liners, which were inserted in short dipole magnets of the SPS accelerator. The electron current measured by stripline monitors connected to the two test liners showed that in this configuration the e-cloud induced by the SPS proton beam is suppressed as efficiently as by the a-C coatings. The different laser parameters employed for treating the two liners led to similar results, demonstrating the robustness of the laser technology.

The work presented in this paper motivates further investigation into the LESS technology, in particular to assess its potential for e-cloud mitigation at cryogenic temperature, including its effect on beam impedance and on static and dynamic vacuum due to roughness and the increased surface area, and to quantify possible particle generation. The engineering goal at this stage is to scale up the demonstrated laboratory-scale process to the automated treatment of larger components using industrial-grade devices, which should allow to greatly improve the treatment speeds. Hence, a collaborative effort with the goal of producing a laser device aimed at in situ treatment of the HL-LHC triplet surfaces has recently been launched, as a first step in investigating large-scale deployment of this technology.

\section{ACKNOWLEDGMENTS}

The authors would like to thank Alexander Lunt (CERN) for the FIB images, and Ivo Wevers and Erik Képeš (CERN) for the water outgassing measurements. The laser surface engineering at the University of Dundee was conducted in house under the aegis of grants and financial support from the Engineering and Physical Sciences Research Council (EPSRC) of the 
United Kingdom (Grant No. EP/I004173/1) and CERN (Grant No. CA6009460). The ASTeC team would like to acknowledge financial support from STFC for the Proof-of-Concept grant for initial development and feasibility study of the LESS technology for reducing SEY. Furthermore, we give our gratitude to the ASTeC management for providing funding for producing the liner laser treatment and for other associated costs. We also would like to thank the Micronanics team for excellent work.

[1] F. Zimmermann, Review of single bunch instabilities driven by an electron cloud, Phys. Rev. ST Accel. Beams 7, 124801 (2004).

[2] M. Tobiyama, J. W. Flanagan, H. Fukuma, S. Kurokawa, K. Ohmi, and S. S. Win, Coupled bunch instability caused by an electron cloud, Phys. Rev. ST Accel. Beams 9, 012801 (2006).

[3] F. Zimmermann, Electron-cloud effects in past and future machines-Walk through 50 years of electron-cloud studies, in Proceedings of the Workshop on Electron-Cloud Effects ECLOUD'12, La Biodola, Isola d'Elba, Italy, 2012 (CERN, Geneva, Switzerland, 2013).

[4] G. Bellodi, O. Bruning, J. Crittenden, G. Rumolo, D. Schulte, X. Zhang, and F. Zimmermann, http://proj-ecloudcode.web.cern.ch/proj-ecloud-code/.

[5] R. Cimino, M. Commisso, D. R. Grosso, T. Demma, V. Baglin, R. Flammini, and R. Larciprete, Nature of the Decrease of the Secondary-Electron Yield by Electron Bombardment and its Energy Dependence, Phys. Rev. Lett. 109, 064801 (2012).

[6] Y. Suetsugu, H. Fukuma, L. Wang, M. T. F. Pivi, A. Morishige, Y. Suzuki, and M. Tsukamoto, Demonstration of electron clearing effect by means of a clearing electrode in high-intensity positron ring, Nucl. Instrum. Methods Phys. Res., Sect. A 598, 372 (2009).

[7] Y. Cai, M. Pivi, and M. A. Furman, Buildup of electron cloud in the PEP-II particle accelerator in the presence of a solenoid field and with different bunch pattern, Phys. Rev. ST Accel. Beams 7, 024402 (2004).

[8] Y. Suetsugu, K. Kanazawa, K. Shibata, and H. Hisamatsu, Continuing study on the photoelectron and secondary electron yield of TiN coating and NEG (Ti-Zr-V) coating under intense photon irradiation at the KEKB positron ring, Nucl. Instrum. Methods Phys. Res., Sect. A 556, 399 (2006).

[9] C. Y. Vallgren, G. Arduini, J. Bauche, S. Calatroni, P. Chiggiato, K. Cornelis, P. C. Pinto, B. Henrist, E. Métral, H. Neupert, G. Rumolo, E. Shaposhnikova, and M. Taborelli, Amorphous carbon coatings for the mitigation of electron cloud in the CERN Super Proton Synchrotron, Phys. Rev. ST Accel. Beams 14, 071001 (2011).

[10] M. Pivi, F. K. King, R. E. Kirby, T. O. Raubenheimer, G. Stupakov, and F. Le Pimpec, Sharp reduction of the secondary electron emission yield from grooved surfaces, J. Appl. Phys. 104, 104904 (2008).
[11] V. Nistor, L. A. González, L. Aguilera, I. Montero, L. Galán, U. Wochner, and D. Raboso, Multipactor suppression by micro-structured gold/silver coatings for space applications, Appl. Surf. Sci. 315, 445 (2014).

[12] Y. Suetsugu, K. Kanazawa, K. Shibata, T. Ishibashi, H. Hisamatsu, M. Shirai, and S. Terui, Results and problems in the construction phase of the SuperKEKB vacuum system, J. Vac. Sci. Technol. A 34, 021605 (2016).

[13] R. Valizadeh, O. B. Malyshev, S. Wang, S. A. Zolotovskaya, W. A. Gillespie, and A. Abdolvand, Low secondary electron yield engineered surface for electron cloud mitigation, Appl. Phys. Lett. 105, 231605 (2014).

[14] http://hilumilhc.web.cern.ch/.

[15] G. Iadarola, E. Metral, and G. Rumolo, CERN Report No. CERN-ACC-2016-0112.

[16] P. C. Pinto, S. Calatroni, H. Neupert, D. Letant-Delrieux, P. Edwards, P. Chiggiato, M. Taborelli, W. Vollenberg, C. Yin-Vallgren, J. L. Colaux, and S. Lucas, Carbon coatings with low secondary electron yield, Vacuum 98, 29 (2013).

[17] G. Arduini, P. Collier, B. Dehning, G. Ferioli, B. Henrist, L. Jensen, J. M. Jiménez, J. M. Laurent, G. Rumolo, K. Weiss, and F. Zimmermann, Electron Cloud Studies and Analyses at SPS for LHC-Type Beams, in Proceedings of the 8th European Particle Accelerator Conference, Paris, 2002 (EPS-IGA and CERN, Geneva, 2002).

[18] G. Tang and A. Abdolvand, Structuring of titanium using a nanosecond-pulsed Nd: YVO4 laser at $1064 \mathrm{~nm}$, Int. J. Adv. Manuf. Technol. 66, 1769 (2013).

[19] N. Chichkov, C. Momma, S. Nolte, E. von Alvensleben, and A. Tunnermann, Femtosecond picosecond and nanosecond laser ablation of solids, Appl. Phys. A 63, 109 (1996).

[20] Z. Lin, L. V. Zhiglilei, and V. Celli, Electron-phonon coupling and electron heat capacity of metals under conditions of strong electron-phonon nonequilibrium, Phys. Rev. B 77, 075133 (2008).

[21] L. V. Zhigileie, Z. Lin, and D. S. Ivanov, Atomistic modeling of short pulse laser ablation of metals: Connections between melting, spallation, and phase explosion, J. Phys. Chem. C 113, 11892 (2009).

[22] R. Valizadeh, O. B. Malyshev, S. Wang, T. Sian, M. D. Cropper, and N. Sykes, Reduction of secondary electron yield for E-cloud mitigation by laser ablation surface engineering, Appl. Surf. Sci. 404, 370 (2017).

[23] R. Valizadeh, O. B. Malyshev, S. Wang, T. Sian, L. Gurran, P. Goudket, M. D. Cropper, and N. Sykes, Low secondary electron yield of laser treated surfaces of copper, aluminum and stainless steel, in Proceedings of IPAC2016, Busan, Korea (JACoW, Geneva, Switzerland, 2016), pp. 1089-1092.

[24] B. Henrist, N. Hilleret, M. Jimenez, C. Scheuerlein, M. Taborelli, and G. Vorlaufer, Secondary electron emission data for the simulation of electron cloud, in Proceedings of the Mini Workshop on Electron Cloud Simulations for Proton and Positron Beams ECLOUD'02, 2002 (CERN, Geneva, Switzerland, 2002).

[25] G. Iadarola and G. Rumolo, Electron cloud in the CERN accelerators (PS, SPS, LHC), in Proceedings of the Workshop on Electron-Cloud Effects ECLOUD'12, La Biodola, Isola d'Elba, Italy, 2012 (CERN, Geneva, Switzerland, 2013). 